\title{
Incomitance of Ocular Rotation Axes in Trochlear Nerve Palsy
}

\author{
KONRAD P. WEBER, ${ }^{a}$ ANTONELLA PALLA,${ }^{a}$ KLARA LANDAU, ${ }^{b}$ \\ THOMAS HASLWANTER, ${ }^{a}{ }^{\text {AND DOMINIK STRAUMANN }}{ }^{a}$ \\ ${ }^{a}$ Department of Neurology, Zurich University Hospital, Zurich, Switzerland \\ ${ }^{b}$ Department of Ophthalmology, Zurich University Hospital, Zurich, Switzerland
}

\begin{abstract}
Strabismus due to palsy of a single muscle in one eye is always incomitant, which is a consequence of Hering's law of equal innervation. We asked whether this law had similar consequences on the orientation of ocular rotation axes. Patients with unilateral trochlear nerve palsy were oscillated about the nasooccipital (= roll) axis $\left( \pm 35^{\circ}, 0.3 \mathrm{~Hz}\right)$, and monocularly fixed on targets on a head-fixed Hess screen. Both the covered and uncovered eyes were measured with dual search coils. The rotation axis of the covered eye (paretic or healthy) tilted more nasally from the line of sight when gaze was directed toward the side of the healthy eye. The rotation axis of the viewing eye (paretic or healthy), however, remained roughly aligned with the line of sight. We conclude that incomitance due to eye muscle palsy extends to ocular rotation axes during vestibular stimulation.
\end{abstract}

KEYWORDS: strabismus; eye movements; vestibuloocular reflex; kinematics; three-dimensional

\section{INTRODUCTION}

Strabismus due to palsy of a single muscle in one eye is always incomitant; that is, the deviation between the two eyes increases when gaze is moved in the pulling direction of the paretic muscle. A direct consequence of Hering's law of equal innervation ${ }^{1}$ is that for a specific position of one eye, the deviation between two eyes is equal, regardless of which eye is covered. ${ }^{2}$ Therefore, the squint angle between the two eyes is smaller when the healthy eye fixes upon a given target (primary deviation) than when the paretic eye fixes upon the very same target (secondary deviation). ${ }^{3}$

In trochlear nerve palsy the force exerted by the superior oblique muscle is reduced. Hence, the line of sight of the covered eye no longer points toward the target, but deviates extorsional-upward or extorsional-downward when the paretic or healthy eye is covered, respectively. As a consequence of Hering's law, this torsional-vertical deviation increases when neural signals associated with ocular counterroll try to activate the paretic superior oblique muscle. Based on this obser-

Address for correspondence: D. Straumann, M.D., Neurology Department, Zürich University Hospital, CH-8091 Zürich, Switzerland. Voice: 41-1-255-5564; fax: 41-1-255-4507.

dominik@neurol.unizh.ch

Ann. N.Y. Acad. Sci. 1004: 347-351 (2003). @ 2003 New York Academy of Sciences. doi: 10.1196/annals.1303.032 
vation, Bielschowsky and Hofmann described the head-tilt test for diagnosing trochlear nerve palsy. ${ }^{4}$

We asked whether Hering's law had similar consequences on the orientation of ocular rotation axes as on the position of the eyes. Specifically, we speculated that in patients with trochlear nerve palsy the angle between the rotation axes of the two eyes were incomitant during Bielschowsky head-tilt testing. To determine the orientations of rotation axes, we used continuous sinusoidal vestibular stimulation about the nasooccipital axis while one eye was covered and the other was fixing upon targets on a Hess screen.

\section{METHODS}

We tested 12 patients (age 15-57 years, 2 female) with non-operated unilateral trochlear nerve palsy. The comparison group consisted of 11 healthy subjects (age 21-40 years, 6 female). The protocol was approved by a local ethics committee and was in accordance with the ethical standards laid down in the Declaration of Helsinki for research involving human subjects.

Subjects were seated upright on a turntable with three servocontrolled motor driven axes (prototype built by Acutronic, Jona, Switzerland). The head was restrained with an individually molded thermoplastic mask (Sinmed BV, Reeuwijk, The Netherlands). Movements of both eyes were recorded with dual search coils in three dimensions ${ }^{5}$ (horizontal, vertical, torsional) on the turntable. Subjects monocularly fixed upon nine laser dots projected on a spherical screen at a distance of $1.4 \mathrm{~m}$, whereas the other eye was covered. The dots were located straight ahead and at eight eccentric head-fixed positions $\left(0^{\circ}\right.$ and $\pm 20^{\circ}$ horizontal and vertical, square grid). During monocular fixation, subjects were oscillated about the nasooccipital (= roll) axis $\left( \pm 35^{\circ}, 0.3 \mathrm{~Hz}\right)$. Eye and chair position signals were digitized with 16-bit accuracy. All data were sampled at $1 \mathrm{kHz}$ and analyzed off-line with Matlab software (The MathWorks, Inc., Natick, MA).

Three-dimensional eye positions in the magnetic coil frame were expressed as rotation vectors. ${ }^{6}$ From these rotation vectors, the corresponding gaze direction was calculated. In addition, the corresponding angular velocity vectors $\vec{\omega}$ were derived to determine "ocular rotation axes," that is, the direction of $\vec{\omega} .^{7}$ The length of $\vec{\omega}$ is proportional to the rotational speed. To relate the ocular rotation axes to the corresponding gaze trajectories on the same Hess screen chart, we projected them stereographically. Data from patients with left-sided trochlear nerve palsies were mirrored to the right side.

\section{RESULTS}

FIGURE 1 summarizes the locations of the rotation axes during monocular fixation of the nine cardinal gaze directions in patients and healthy subjects. The paretic right eyes of the patients, when covered (FIG. 1B, gray ellipses), showed rotation axes that, on average, were shifted nasally compared with the eyes of healthy subjects (black ellipses). This horizontal deviation increased with adduction of the covered paretic eye. The healthy left eyes of patients, when covered (FIG. 1A, gray ellipses), showed 

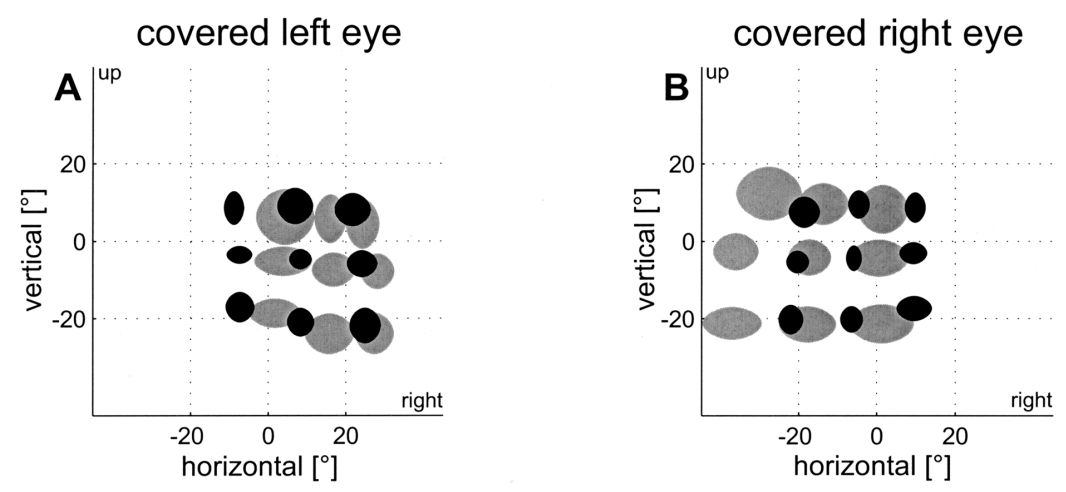

FIGURE 1. Orientation of ocular rotation axes (stereographic projection onto Hess screen). Ellipses: average (center) \pm 1 SD (horizontal and vertical radius). Healthy subjects (black); patients (gray). Intersections of dotted lines indicate location of visual targets. (A) Left eye during right eye viewing. (B) Right eye during left eye viewing.

a nasal shift of the rotation axes as well, but, in contrast to the paretic eyes, the deviation increased with abduction. The rotation axes of the viewing eyes in patients and healthy subjects matched closely (not shown); that is, the rotation axes in both groups of subjects were near the line of sight, when fixation was maintained by the respective eye.

To test whether the eye position-dependent angle between the rotation axes of both eyes follows the same principle as the incomitant squint angle, we used the graphical method to analyze strabismus proposed by Zee et al. ${ }^{2}$ but plotted the absolute horizontal angle of ocular rotation axes of both eyes, instead of their horizontal gaze position, against each other (FIG. 2). Data points of axes moving in parallel would lie on a line with zero intercept and a slope of one (dashed line). Data points below this line represent convergent axes and data points above the line represent divergent axes. A slope of one always indicates that axes are comitant; otherwise, they are incomitant. Data points of healthy subjects with either eye covered were located on a regression line with a slope of almost one, indicating comitance (FIG. 2, squares). Thus, analogous to binocular eye positions, binocular axes were comitant in healthy subjects, indicating that Hering's law of equal innervation extends to the orientation of rotation axes. Consistent with the fact that axes normally show a slight convergence, ${ }^{8}$ data points were situated below the dashed line.

The slopes of regression lines in patients (triangles: average of pooled data from fixations along the horizontal meridian) were larger than one, demonstrating incomitance between the rotation axes of both eyes. As in healthy subjects, the fitted lines through the data with either eye covered (filled: paretic right eye covered = primary axis deviation, open: healthy left eye covered = secondary axis deviation) were similar. In other words, for a given axis orientation of one eye, the angle between the ocular rotation axes was almost the same, independent of which eye was covered. Note, however, that, for a given orientation of an ocular rotation axis in one eye, the deviation of the axis of the fellow eye was larger when the former eye was the 


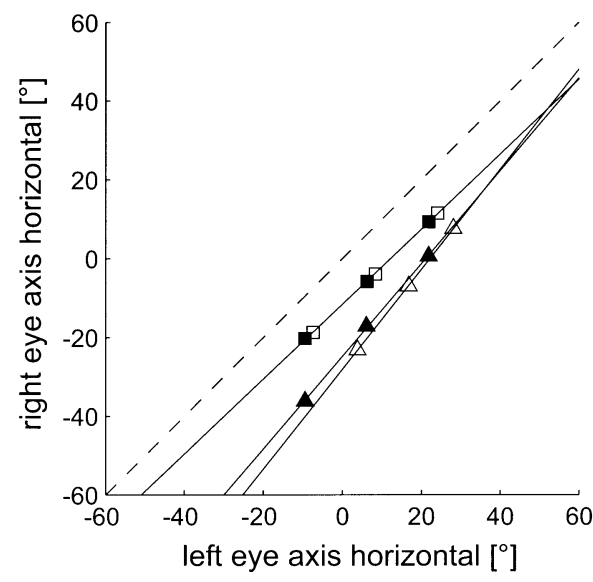

FIGURE 2. Comitance and vergence of ocular rotation axes during fixations along horizontal meridian. Orientations of rotation axes of both eyes plotted against each other. Triangles: Average data in patients; squares: average data in healthy subjects; open symbols: right eye viewing condition; filled symbols: left eye viewing condition; dashed line: absolute comitance and no vergence between both eyes; solid lines: first-order linear regression. Data points below dashed line: convergence. Slope $>1$ : increasing convergence of axes with left gaze.

healthy one. Thus, the primary axis deviation was larger than the secondary axis deviation. This is exactly opposite of what one observes for eye positions with the primary squint angle being smaller than the secondary squint angle.

\section{DISCUSSION}

Our data reveal that in patients with trochlear nerve palsy Hering's law has similar consequences on the orientation of ocular rotation axes as on eye positions. During torsional vestibular stimulation about the nasooccipital axis, ocular rotation axes are incomitant. In the covered condition the rotation axis of both the paretic and healthy eye moves nasally; its deviation from the rotation axis of the viewing eye, however, increases when the patient looks in the direction of the healthy eye.

A detailed geometrical explanation for the incomitance of rotation axes will be given elsewhere (Weber et al., in preparation). Intuitively, however, this phenomenon can be understood for the situation when the paretic eye is covered: the larger the vertical component of the superior oblique (SO) muscle is in a given eye position, the more a paresis will lead to a deviation of the ocular rotation axis from the line of sight. Because the vertical component of the SO muscle pulling direction increases with adduction, we can expect a larger deviation of the ocular rotation axis in this gaze direction.

To explain the incomitance of rotation axes when the healthy eye is covered, one must apply Hering's law of equal innervation to conjugate torsion. This can be done by assuming equal distribution of innervation between two muscle pairs, not just 
single muscles. Specifically, superior oblique and superior rectus muscles of one eye are yoked with inferior oblique and inferior rectus muscles of the other eye, respectively. If the SO muscle becomes paretic and the subject fixates with the ipsilateral eye, there will be hyperinnervation in the healthy eye along a vector parallel the vector of the paretic SO. This in turn will lead to incomitance of ocular rotation axes along the horizontal direction (geometrical details: Weber et al., in preparation).

In conclusion, the concept of incomitance in the presence of eye muscle palsy includes not only binocular positions, but also binocular rotation axes during concurrent vestibular stimulation.

\section{ACKNOWLEDGMENTS}

The authors thank Chris Bockisch, Albert Züger, and Tanja Schmückle for their assistance. This work was supported by Swiss National Science Foundation 3251938.97 SCORE A/31-63465.00 and Betty and David Koetser Foundation for Brain Research.

\section{REFERENCES}

1. Hering, E. 1868. Die Lehre vom binocularen Sehen. Verlag von Wilhelm Engelmann, Leipzig.

2. ZEE, D.S., F.C. CHU, L.M. OPTICAN, et al. 1984. Graphic analysis of paralytic strabismus with the Lancaster red-green test. Am. J. Ophthalmol. 97: 587-592.

3. von NoORDEN, G.K. 1996. Binocular Vision and Ocular Motility. 5th edit. Mosby. St. Louis, MO.

4. BIELSCHOWSKY, A. \& F.B. HofmANN. 1900. Die Verwerthung der Kopfneigung zur Diagnostik von Augenmuskellähmungen aus der Heber- und Senkergruppe. [The evaluation of head inclination for the diagnosis of pareses of ocular muscles from the group of elevators and depressors.]. Graefes Arch. Ophthalmol. 51: 174-185.

5. BERGamin, O., D.S. ZEE, D.C. RobERTS, et al. 2001. Three-dimensional Hess screen test with binocular dual search coils in a three-field magnetic system. Invest. Ophthalmol. Visual Sci. 42: 660-667.

6. HAUSTEIN, W. 1989. Considerations on Listing's law and the primary position by means of a matrix description of eye position control. Biol. Cybern. 60: 411-420.

7. Hepp, K. 1990. On Listing's law. Commun. Math. Phys. 132: 285-292.

8. Bergamin, O. \& D. StRAumAnN. 2001. Three-dimensional binocular kinematics of torsional vestibular nystagmus during convergence on head-fixed targets in humans. J. Neurophysiol. 86: 113-122. 